\title{
Interaktionen zwischen respiratorischem Mikrobiom und Epithelzellen formen Immunität in der Lunge
}

\author{
Rachele Invernizzi ${ }^{a} \quad$ Clare M. Lloyd $^{a}$ Philip L. Molyneaux ${ }^{a}$ b \\ aInflammation, Repair and Development Section, National Heart and Lung Institute, Imperial College, London, UK; \\ ${ }^{b}$ Department of Respiratory Medicine, Interstitial Lung Disease Unit, Royal Brompton Hospital, London, UK
}

\author{
Schlüsselwörter \\ Immunität · Lunge · Mukosale Immunologie · Respiratorisches \\ Epithel $\cdot$ Respiratorisches Mikrobiom
}

\section{Zusammenfassung}

Das respiratorische Epithel stellt eine physische Grenze zur äußeren Umgebung dar und fungiert als erste Verteidigungslinie gegen potenziell schädliche Umweltreize wie Mikroben und Allergene. Die Epithelzellen in der Lunge gelten jedoch zunehmend auch als aktive Effektoren der Abwehr von Erregern, die zur angeborenen und adaptiven Immunfunktion im unteren Respirationstrakt beitragen. Diese Zellen exprimieren ein breites Spektrum an Pattern-Recognition-Rezeptoren, die spezifisch für Strukturmotive der Erreger und des Wirts sind. Mit Hilfe moderner molekularer Verfahren konnte die Komplexität des Mikrobioms im unteren Respirationstrakt aufgedeckt werden. Das Wechselspiel zwischen Mikrobiota und respiratorischem Epithel ist der Schlüssel zum Verständnis der Aufrechterhaltung einer stabilen Immunhomöostase. Ein Verlust der Epithelintegrität nach Exposition gegenüber einer Infektion kann bei anfälligen Personen eine Entzündung auslösen und letztlich zu einer Lungenerkrankung führen. In der vorliegenden Arbeit erörtern wir den aktuellen Erkenntnisstand in Bezug auf die molekularen und zellulären Mechanismen, über die das Lungenepithel mit dem Lungenmikrobiom interagiert und so eine Immunität in der Lunge formt. Im Mittelpunkt stehen insbesondere die Interaktionen zwischen dem Lungenmikrobiom und den Zellen der leitenden Atemwege bei der Modulierung der Immunzellregulation sowie die Frage, wie Defekte in der Barrierestruktur und -funktion letztlich zu einer Lungenerkrankung führen können. Diese Interaktionen zu verstehen, ist bei der Suche nach wirksameren Therapien bei Atemwegserkrankungen von fundamentaler Bedeutung.

(c) 2020 Die Autoren

\section{Die Epithelbarriere}

Der menschliche Respirationstrakt ist die Haupteintrittspforte für eine breite Palette von Luftkeimen und Schwebeteilchen, u.a. Viren, Bakterien und Pilze, welche über diese stetig einwandern und dort auch eliminiert werden. Die Lunge ist fortwährend einer Vielzahl von Mikroorganismen ausgesetzt, von denen einige persistieren und den Respirationstrakt besiedeln können. Diese Mikroben in den unteren Atemwegen stellen das Lungenmikrobiom dar, das sich in seiner Zusammensetzung von der Mikrobiota in
Mund- und Nasenhöhle unterscheidet. Die Lungenoberfläche ist eine kontinuierliche Schicht aus Epithelzellen, die eine physische und biologische Barriere für eingeatmete Substanzen und Pathogene darstellen. Das spezialisierte respiratorische Epithel ist für eine Aufrechterhaltung der Immunhomöostase erforderlich, und eine epitheliale Dysfunktion spielt eine Rolle bei der Entwicklung zahlreicher entzündlicher Erkrankungen der Atemwege und der Lunge [1]. Der Respirationstrakt ist ein komplexes Organsystem, dessen primäre Funktion der Austausch von Sauerstoff und Kohlendioxid ist. Er wird unterteilt in den oberen Respirationstrakt, 


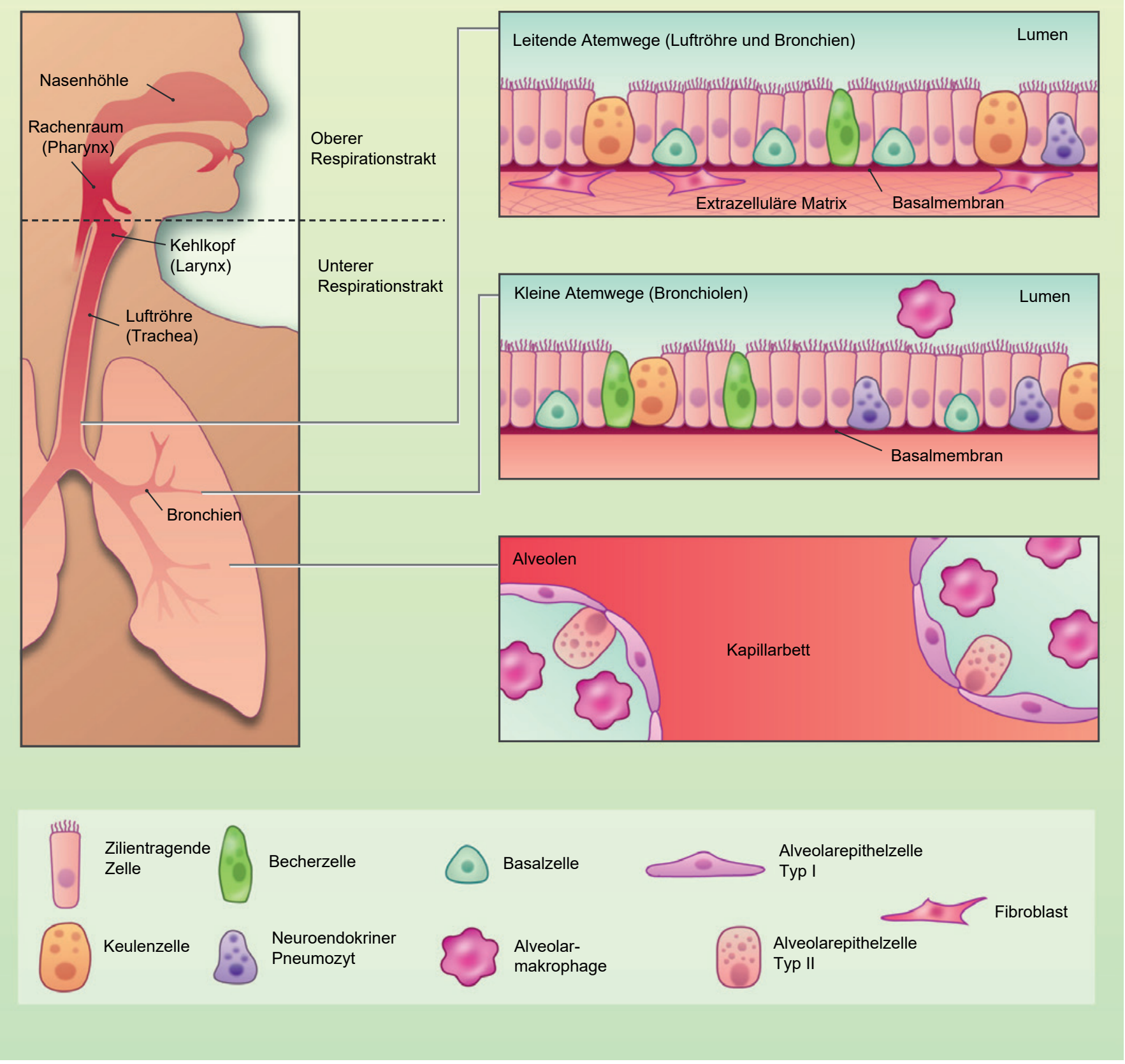

Abb. 1. Zusammensetzung des gesunden respiratorischen Epithels. Der Respirationstrakt des Menschen wird unterteilt in den oberen Respirationstrakt, der Nasenhöhle, Rachen und Kehlkopf umfasst, sowie den unteren Respirationstrakt, der die leitenden Atemwege (Luftröhre und Bronchien), die Bronchiolen und die Alveolen umfasst. Das Epithel der Luftröhre und großen Atemwege besteht aus zilientragenden Zellen («Flimmerepithel»), Mukus (Schleim) produzierenden Becherzellen, neuroendokrinen Zellen und undifferenzierten Basalzellen. Stromazellen, u.a. Fibroblasten, bilden die extrazelluläre Matrix und modulieren den Umsatz der respiratorischen Epithelzellen. Die Alveolen bestehen aus Alveolarmakrophagen sowie Alveolarepithelzellen des Typs I und Typs II.

der Nasenhöhle, Rachen und Kehlkopf umfasst, sowie den unteren Respirationstrakt, der die leitenden Atemwege (Luftröhre und Bronchien), die Bronchiolen und die Alveolen umfasst. Die Atemwege des Menschen besitzen eine Oberfläche von rund 70 $\mathrm{m}^{2}$, auf der sich eine Vielzahl von Bakteriengemeinschaften findet. Die höchste Bakterienlast lässt sich dabei im oberen Respirationstrakt beobachten [2]. Der Respirationstrakt erstreckt sich über verschiedene anatomische Bereiche, und die dort jeweils be- findlichen Epithelzellen unterscheiden sich sowohl in ihrer Zusammensetzung als auch in ihrer Struktur. Dies spiegelt die unterschiedlichen Funktionen der Epithelzellen im Respirationstrakt wider, welche die leitenden Atemwege und die Alveolarbereiche der Lunge auskleiden (Abb. 1).

Im unteren Respirationstrakt bildet das respiratorische Epithel eine hoch wirksame Barriere und fungiert als erste Verteidigungslinie gegen potenziell schädliche Umweltreize wie etwa Mikroben 
und Allergene [1]. Das pseudostratifizierte Epithel der leitenden Atemwege besteht primär aus zilientragenden Zellen, Mukus produzierenden Becherzellen, neuroendokrinen Zellen und Basalzellen, die eine oberflächenaktive Substanz, das Surfactant, sezernieren [1]. Es stellt den ersten Bereich der Interaktion mit eingeatmeten Umweltsubstanzen dar, und seine Aufgabe besteht in einer wirksamen mukoziliären Beseitigung («Clearance») von Partikeln und Mikroben [3]. Zilientragende Zellen besitzen am apikalen Zellpol sogenannte Zilien (Flimmerhärchen), die sich koordiniert bewegen, um eingeatmete Partikel und Mukus aus den Atemwegen hinaus zu befördern. Becherzellen und sekretorische Zellen fangen hingegen eingeatmete Partikel und Mikroorganismen ab. Bei den Basalzellen handelt es sich um Vorläuferzellen, die als gewebsständige Stammzellen für die Luftröhre und die proximalen Atemwege fungieren. Diese Zellen können sich selbst erneuern und das pseudostratifizierte Epithel während der Homöostase und nach einer Schädigung wieder neu auskleiden [4]. In der Luftröhre und den großen Atemwegen finden sich Stromazellen, wie etwa interstitielle Fibroblasten, die zur Regulation der regenerativen Reaktion des respiratorischen Epithels nach Schädigungen beitragen [5]. Im Gegensatz dazu sind die Alveolarflächen in der peripheren Lunge mit flachen Alveolarepithelzellen (Pneumozyten) Typ 1 (AEC1) ausgekleidet, die eine gleichmäßige Zellschicht bilden und auf den Gasaustausch spezialisiert sind. Die kubischen Alveolarepithelzellen Typ 2 (AEC2) fungieren als Vorläuferzellen und bilden Surfactant, das die Oberflächenspannung senkt, um das Kollabieren der Alveolen bei der Atmung zu verhindern [6, 7]. Ein gemeinsames Merkmal dieser Zelltypen sind die Zell-ZellVerbindungen (sogenannte Tight Junctions) auf der apikalen Oberfläche, die für die Adhäsions- und Barrierefunktion des Epithels von zentraler Bedeutung sind [8]. Diese Tight Junctions sorgen dafür, dass die Zellen aneinander haften und eine regelmäßige, undurchdringliche Barriere bilden. Dieser intrazelluläre Adhäsionskomplex besteht aus miteinander verbundenen Proteinen und Rezeptoren, u.a. Zonula occludens (ZO)-1, -2 und -3, Occludinen, Claudinen, Transmembran- und Zelladhäsionsmolekülen. Tight Junctions steuern die parazelluläre Permeabilität. Unmittelbar unterhalb von diesen liegen die Adherens Junctions, aus $\beta$-Catenin und E-Cadherin bestehende Adhäsionsverbindungen, die die aneinander angrenzenden Zellen mechanisch verbinden und deren Proliferation und Differenzierung initiieren [8, 9]. Vorkommen und Funktion dieser Komplexe können durch Zelldifferenzierung und die Exposition gegenüber verschiedenen Stimuli wie Allergenen und Schadstoffen beeinflusst werden. Bei Krankheitszuständen können sie verändert sein und so zur Immunpathologie beitragen $[10,11]$.

\section{Das Lungenmikrobiom in Gesundheit und Krankheit}

Die lange Zeit vertretene, jedoch unzutreffende Theorie einer Sterilität der Lunge außerhalb einer klinischen Infektion bedeutet, dass in der Vergangenheit nur wenige Arbeiten die Rolle des Lungenmikrobioms bei chronischen Lungenerkrankungen untersucht haben. Fortschritte im Bereich der Hochdurchsatz-Sequen- zierung haben dieser Vorstellung den Nimbus genommen und den Nachweis erbracht, dass die Epitheloberflächen des menschlichen Respirationstrakts von einer komplexen und dynamischen Mikrobiota, dem sogenannten Lungenmikrobiom, besiedelt sind, das sowohl bei Gesundheit als auch bei Krankheit eine Rolle spielt [14]. Der am häufigsten verwendete Ansatz zur Untersuchung von Bakteriengemeinschaften macht sich die Hochdurchsatz-Sequenzierung von Amplicons des rRNA-Gens 16S zu Nutze, einem hoch konservierten Lokus im Bakteriengenom mit spezifischen variablen Regionen, die zur phylogenetischen Klassifikation herangezogen werden können [15]. Sequenzierungsstudien zeigen ausgeprägte Unterschiede im Lungenmikrobiom zwischen Gesundheit und Krankheit, und es zeichnet sich zunehmend ab, dass das Lungenmikrobiom möglicherweise nicht nur zur Erklärung der Pathogenese der Erkrankungen beitragen, sondern auch als prognostischer Marker und therapeutisches Target dienen könnte $[16,17]$.

In gesundem Zustand besitzt die Lungenmikrobiota eine niedrige Dichte, weist dabei aber eine auffällige Diversität miteinander interagierender Mikroorganismen auf. Die Atemwegsmikrobiota der gesunden Lunge besteht in erster Linie aus Bacteroidetes, Firmicutes und Proteobacteria. Zu den herausragenden Gattungen in den unteren Atemwegen zählen Prevotella, Veillonella und Streptococcus [21]. Die Zusammensetzung des Lungenmikrobioms wird durch drei Faktoren bestimmt: Die Einwanderung von Mikroben, die Elimination von Mikroben und die relative Reproduktionsrate der beteiligten Organismen. Die Einwanderung von Mikroben in die Lunge erfolgt im Wesentlichen über eine Inhalation luftgetragener Bakterien, unmittelbare Verteilung über Schleimhautoberflächen und Mikroaspiration [14, 22]. Die Ähnlichkeiten bezüglich der Zusammensetzung der Gemeinschaften zwischen Mundhöhlen- und Lungenmikrobiota lassen darauf schließen, dass die Mikroaspiration den vorherrschenden Einwanderungsweg darstellt [14, 23]. Die Elimination von Mikroorganismen ist ein aktiver Vorgang und wird durch mukoziliäre Clearance, Husten und Immunabwehrmechismen des Wirts erreicht [24]. Die für das mikrobielle Wachstum im Respirationstrakt erforderlichen Umgebungsbedingungen (z.B. pH-Wert, Temperatur, Verfügbarkeit von Nährstoffen, Sauerstoffpartialdruck und Aktivierung von Wirtsentzündungszellen) sind heterogen, und in den einzelnen Abschnitten der gesunden Lunge lassen sich erhebliche Unterschiede beobachten. Bei einer Lungenerkrankung ist das Gleichgewicht zwischen mikrobieller Einwanderung und Elimination gestört, was zu Veränderungen in der Lungmikrobiota führt. Hierbei übernehmen Bakterien, die kompetitive Vorteile besitzen, zunehmend eine vorherrschende Stellung [14] (Tab. 1). Das übermäßige Wachstum bestimmter Bakterienarten führt zu einer Abnahme der Gesamtvielfalt und geht einher mit der Progression chronischer Lungenerkrankungen wie Asthma, chronisch-obstruktiver Lungenerkrankung (COPD), zystischer Fibrose (CF) und idiopathischer Lungenfibrose (IPF) [16, 17, 19, 20, 25]. Des Weiteren wurde in diesen Studien bei einer Vielzahl chronischer Atemwegserkrankungen ein Zusammenhang zwischen mikrobieller Dysbiose und erhöhter Morbidität bzw. Mortalität festgestellt. 
Tab. 1. Studienübersicht zur Veranschaulichung von Veränderungen des respiratorischen Mikrobioms bei chronischen Lungenerkrankungen

\begin{tabular}{|c|c|c|c|c|}
\hline Erkrankung & Taxonomische Veränderungen auf Gattungsebene ${ }^{1}$ & Probentyp & $\begin{array}{l}\text { Kohorten- } \\
\text { größe }\end{array}$ & [Ref.] \\
\hline \multirow[t]{3}{*}{ Asthma } & Erhöhte Häufigkeit von Haemophilus & Bronchoskopie & 11 & {$[16]$} \\
\hline & $\begin{array}{l}\text { Erhöhte Häufigkeit von Haemophilus, } \\
\text { Neisseria, Fusobacterium und Porphyromonas }\end{array}$ & Bronchoskopie & 42 & {$[29]$} \\
\hline & Erhöhte Häufigkeit von Streptococcus & Nasopharyngeal & 234 & {$[30]$} \\
\hline \multirow[t]{2}{*}{ COPD } & Erhöhte Häufigkeit von Lactobacillus & Lungengewebe & 24 & {$[17]$} \\
\hline & $\begin{array}{l}\text { Erhöhte Häufigkeit von Fusobacteria, } \\
\text { Leptotrichia und Fusobacterium }\end{array}$ & $\mathrm{BAL}$ & 32 & {$[31]$} \\
\hline \multirow[t]{3}{*}{ CF } & Erhöhte Häufigkeit von Burkholderia, Streptococcus und Staphylococcus & Sputum & 23 & {$[25]$} \\
\hline & $\begin{array}{l}\text { Erhöhte Häufigkeit von Pseudomonas, Staphylococcus, Stenotrophomonas und } \\
\text { Achromobacter }\end{array}$ & Sputum & 17 & {$[32]$} \\
\hline & Erhöhte Häufigkeit von Staphylococcus, Streptococcus und Pseudomonas & $\mathrm{BAL}$ & 95 & {$[33]$} \\
\hline \multirow[t]{3}{*}{ IPF } & Erhöhte Häufigkeit von Haemophilus, Streptococcus, Neisseria und Veillonella & $B A L$ & 65 & {$[19]$} \\
\hline & Erhöhte Häufigkeit von Staphylococcus und Streptococcus & BAL & 55 & {$[20]$} \\
\hline & $\begin{array}{l}\text { Erhöhte Häufigkeit von Streptococcus, Prevotella, Veillonella, Haemophilus und } \\
\text { Pseudomonas }\end{array}$ & $\mathrm{BAL}$ & 35 & [26] \\
\hline
\end{tabular}

BAL, Bronchoalveoläre Lavage; CF, Zystische Fibrose; COPD, Chronisch-obstruktive Lungenerkrankung; IPF, Idiopathische pulmonale Fibrose; ${ }^{1}$ Veränderungen in der Mikrobiota bei Patienten im Vergleich zu Kontrollen.

Es gibt Belege dafür, dass über die bakterielle Komponente des Mikrobioms hinaus andere nicht bakterielle Organismen, u.a. Viren und Pilze, möglicherweise eine wichtige Rolle für Gesundheit und Krankheit spielen. Neue Evidenzdaten lassen darauf schließen, dass das Lungenvirom und Lungenmykobiom erheblichen Einfluss auf den klinischen Ausgang chronischer Atemwegserkrankungen wie Asthma, COPD, CF und IPF haben [27, 34, 35]. Darüber hinaus wird zunehmend anerkannt, dass Atemwegsviren eine Hauptursache für akute Exazerbationen chronischer Lungenerkrankungen darstellen und dass die Wirtsantwort auf Virusinfektionen bei diesen Erkrankungen fehlreguliert ist. So sprechen Untersuchungen beispielsweise für eine mechanistische Rolle von Viren bei Auftreten und Fortschreiten der IPF. Die Familie des humanen Herpesvirus (HHV), zu der das Zytomegalievirus, Epstein-Barr-Virus, HHV-7 und HHV-8 gehören, findet erhebliche Beachtung als ätiologischer bzw. exazerbierender Faktor bei IPF [36]. Bei Asthma ist eine Infektion mit Atemwegsviren ein Hauptauslöser für Giemen bei Kleinkindern sowie für exazerbierendes Asthma bei älteren Kindern. Zu den Infektionserregern, die mit Giemen in Zusammenhang gebracht werden, zählen das respiratorische Synzytialvirus (RSV), das humane Rhinovirus, das Parainfluenza- und Coronavirus sowie andere Viren [37]. Ungeachtet der Tatsache, dass man über das Mykobiom der Lunge weit weniger weiß als über das bakterielle Mikrobiom, wurden in den letzten Jahren spezifische Pilze bei Atemwegserkrankungen nachgewiesen $[38,39]$. Darüber hinaus konnte gezeigt werden, dass kommensale Pilze das Immunsystem beeinflussen und außerdem bakterielle Gemeinschaften modulieren und so zur Wiederherstellung der bakteriellen Mikrobiota nach einer Be- handlung mit Antibiotika beitragen [40, 41]. Auch wenn die Zahl der Studien zur Zusammensetzung und Funktion der Virus- und Pilzgemeinschaften in der Lunge zunimmt, steckt die Forschung in diesem Bereich noch in den Kinderschuhen. Weitere mechanistische Arbeiten sind erforderlich, um neue Einblicke in die Rolle dieser Gemeinschaften bei der Pathogenese von Lungenerkrankungen zu erzielen.

\section{Ein homöostatisches Gleichgewicht zwischen der Lunge und ihrer Mikrobiota}

Die Epithelzellen in den Atemwegen stehen in ständigem Kontakt mit der Umwelt, und dieses Wechselspiel ist ein entscheidender Faktor für die Aufrechterhaltung einer stabilen Homöostase [42]. Die drei wesentlichen Komponenten, die zur Barrierefunktion des respiratorischen Epithels beitragen sind: die mukoziliäre Clearance, durch die inhalierte Mikroben und noxische Stimuli in den Atemwegen abgefangen und abtransportiert werden, intrazelluläre [SAB3] Tight und Adherens Junctions, welche die parazelluläre Permeabilität des Epithels regulieren sowie sezernierte antimikrobielle Peptide, die eingeatmete Krankheitserreger eliminieren [1]. Die physiologischen Merkmale des Respirationstrakts sind der Schlüssel für die Erhaltung eines Gleichgewichts zwischen der Lunge und ihrer Mikrobiota. Das Lungenepithel setzt sich aus anderen Zelltypen zusammen und unterscheidet sich von dem Epithel, das den oberen Respirationstrakt bis zu den Alveolen auskleidet [43]. In den großen Atemwegen enthalten die submukösen Drüsen den Mukus und die serösen Zellen, die für die Produktion eines Großteils des Schleims in den Atemwegen 
verantwortlich sind [44]. In den Bronchiolen wird Mukus von Becher- und Keulenzellen produziert, während in den Alveolen AEC1 und AEC2 Surfactant sezernieren [45-47]. Mukus ist eine Art Gel, das hauptsächlich aus Wasser und glykosylierten Proteinen, z.B. Muzinen, besteht [48]. Muzine sind die großen Glykoproteinbestandteile des Schleims in den Atemwegen [49]. Die vorherrschenden Gel-bildenden Muzine in den Atemwegen des Menschen sind MUC5AC, das in den Becherzellen, sowie MUC5B, das in den submukösen Drüsen produziert wird [50, 51]. Mukus ist eine wichtige ökologische Nische für die Mikrobiota. Er fördert das gesunde Wechselspiel zwischen Mikroorganismen und Epithelzellen durch die Bildung einer dünnen, mobilen Schicht, die ihrerseits durch die periziliäre Schicht, welche die Zilien bedeckt, gestützt wird $[49,52]$. Bei Gesunden bietet die Schleimschicht einen effektiven Schutz gegen Schädigungen des Epithels. Allerdings geht eine übermäßige Mukusproduktion mit einer erhöhten Anfälligkeit für Virusinfektionen und Atemwegsallergien einher und trägt bei verschiedenen Erkrankungen wie Pneumonie, Asthma, COPD und CF zur Obstruktion bei $[53,54]$. Diese Obstruktion behindert die Zilien in ihrer Fähigkeit, Mukus aus den Lungen abzutransportieren und begünstigt bestimmte bakterielle Erreger, die in Mukus gedeihen und sich in dieser blockierten Nische ansiedeln [55]. Des Weiteren ist bei chronischen Lungenerkrankungen häufig eine Mikroaspiration zu beobachten, und es zeigt sich, dass begleitend bei chronischen Atemwegserkrankungen oftmals eine gastroösophageale Refluxkrankheit (GORD) auftritt. Auch wenn sich eine GORD in der Regel auf die untere Speiseröhre beschränkt, kann es bei manchen Personen zu einer Mikroaspiration von Mageninhalt in die Lunge kommen [56]. Eine Aspiration kann zu erhöhten Gallensäurewerten in der Lunge führen und so die Bildung mikrobieller Mikrofilme anregen. Diese bestehen aus Zellen von Mikroorganismen, die, eingebettet in eine extrazelluläre Matrix, strukturierte Gemeinschaften bilden [57]. Biofilme von Bakterien und Pilzen haben sich als Virulenzmechanismus von opportunistischen Erregern wie etwa Pseudomonas aeruginosa und Staphylococcus-Spezies erwiesen $[58,59]$. Ein Kennzeichen von Biofilmen ist ihre bemerkenswerte antimikrobielle Toleranz, die es ihnen erlaubt, im Wirt zu persistieren und seinem Immunsystem zu entgehen [59]. Daher können GORD-bedingte Gallensäure und ein nachfolgendes Biofilmwachstum auf Wirtsgewebe zu einer chronischen Atemwegsinfektion beitragen. Ferner könnte eine direkte Besiedlung der Atemwege mit Bakterien der Darmflora ein Faktor sein, der die Interaktion zwischen Epithel und Mikrobiom beeinflusst [60].

Die intrazellulären Tight und Adherens Junctions ermöglichen stabile adhäsive Kontakte zwischen benachbarten Epithelzellen [61]. Tight Junctions sind die Hauptregulatoren der parazellulären Permeabilität, während die Adherens Junctions benachbarte Zellen mechanisch verbinden und die Bildung und Reifung von Zell-Zell-Kontakten initiieren [8,9]. Im Krankheitsfall ist die Barrierefunktion gestört, was zu einer verstärkten Durchlässigkeit des Epithels und zum Eindringen von Pathogenen führt [10, 11]. Bei einer Infektion sind Epithelzellen in der Lage, mit der Freisetzung von humoralen Faktoren, z.B. antimikrobiellen Pep- tiden, zu reagieren, welche eine antimikrobielle Breitbandwirkung besitzen. Eine Reihe dieser Faktoren wird als Antwort auf die krankheitsbedingte mikrobielle Dysbiose hochreguliert [62]. Defensine sind die am häufigsten vorkommenden antimikrobiellen Peptide und umfassen $\alpha$-Defensine in neutrophilen Granulozyten und $\beta$-Defensine in Epithelzellen. Die humanen $\beta$-Defensine 1, 2, 3 und 4 werden verbreitet auf respiratorischen Epithelzellen exprimiert und fungieren dort als muköse Verteidigungslinie, indem sie eine Vielzahl verschiedener Erreger von Atemwegserkrankungen attackieren. Ihre Expression im respiratorischen Epithel kann konstitutiv oder durch bakterielle Produkte oder proinflammatorische Zytokine induzierbar sein [63]. Die Verfügbarkeit von Nährstoffen in jeder spezifischen Nische spielt ebenfalls eine Rolle bei der Aufrechterhaltung und Zusammensetzung des Lungenmikrobioms. Wirtsverbindungen wie Muzine, Zytokine, Defensine und Lactoferrine stehen in der Lunge vorkommenden Bakterien als Nährstoffquelle zur Verfügung [1, 64]. Einfluss auf die mikrobiellen Wachstumsraten haben ebenfalls Veränderungen des Sauerstoffpartialdrucks. In den verschiedenen Lungenabschnitten finden sich erhebliche Unterschiede im Sauerstoffpartialdruck, die sich auf die Bakterienproliferation auswirken und so die Populationsdynamik verändern können [65]. Bei chronischen Lungenerkrankungen befördert eine erhöhte Schleimproduktion das Bakterienwachstum und führt zur Bildung von Bereichen mit niedriger Sauerstoffkonzentration und hohen Temperaturen, was die Selektion und Erhaltung bestimmter Bakterien begünstigt [14]. Insgesamt haben diese Unterschiede bezüglich der zellulären Funktion und der physiologischen Eigenschaften der Lunge (wie Temperatur, Mukus, Surfactant und Sauerstoffpartialdruck) wesentlichen Einfluss auf die Etablierung und Persistenz bakterieller Gemeinschaften.

\section{Das respiratorische Epithel als Sensor für das Vorliegen von Mikroorganismen}

Das respiratorische Epithel stellt nicht nur eine strukturelle Barriere dar, sondern ist auch von wesentlicher Bedeutung für die angeborene Immunabwehr des Wirtes. Respiratorische Epithelzellen exprimieren verschiedene Pattern-Recognition-Rezeptoren (PRRs) und sezernieren antimikrobielle Moleküle und Muzine, die zur Abwehr eindringender Pathogene beitragen [3]. PRRs sind von Genen der Keimbahnzellen kodierte Rezeptoren, die konservierte Pathogen-assoziierte molekulare Muster (PAMPs) oder Zellschädigung-assoziierte molekulare Muster erkennen [66-68]. Diese Transmembranrezeptoren können unabhängig von der adaptiven Immunantwort aktiviert werden und lassen sich in vier Klassen einteilen: Toll-like-Rezeptoren (TLRs), RIG-I-ähnliche Rezeptoren (RLRs) zytoplasmatischer Proteine, NOD-like-Rezeptoren (NLRs) und C-Typ-Lektin-Rezeptoren (CLRs) [69].

Bislang wurden 10 TLRs (TLR1 - TLR10) im menschlichen Genom identifiziert. Sie sind durch sich wiederholende leucin-reiche Sequenzen (leucine-rich repeats) am N-Terminus, eine Transmembranregion und eine zytoplasmatische Toll/IL-1-Rezeptor
244

Kompass Pneumol 2020;8:240-250 DOI: $10.1159 / 000510786$ 

(TIR)-Homologie-Domäne gekennzeichnet, die die Signalweiterleitung über MyD88 und TRIF (TIR-domain-containing adapterinducing interferon- $\beta$ ) vermittelt [70]. Toll-like-Rezeptoren spielen eine wichtige Rolle bei der Erkennung von Pathogenen und bei der angeborenen Immunität. Die Hauptfunktion von TLRs bei Säugetieren besteht in der Erkennung spezifischer PAMPs, u.a. von Bestandteilen mikrobieller Zellwände (TLR1, TLR2, TLR4 und TLR6), Flagellin (TLR5), einzelsträngiger oder doppelsträngiger Virus-RNA (TLR3, TLR7 und TLR8) oder CpG-haltiger DNA (TLR9) [71-74]. TLR3, 7, 8 und 9 finden sich insbesondere in Endosomen und detektieren nach der Endozytose die Nukleinsäuren von Pathogenen [75-79]. TLR10 schließlich wird bei Menschen exprimiert, aber sein spezifischer Ligand ist noch unbekannt [80]. Die Signalübertragung über TLRs ist mit der Aktivierung und Expression von Wirtszytokinen, Chemokinen und antimikrobiellen Peptiden von entscheidender Bedeutung für eine frühe Entzündungsantwort auf Bakterien. TLRs finden sich auf Immun- und Entzündungszellen. In Anbetracht der Tatsache, dass Zellen des respiratorischen Epithels in Kontakt mit verschiedenen potenziellen Pathogenen kommen, ist die Expression von TLRs jedoch auch für eine Immunität in den Atemwegen relevant. Tatsächlich exprimieren Epithelzellen in den menschlichen Bronchien die Mehrzahl der TLRs, wobei TLR2, TLR3, TLR5 und TLR6 am stärksten exprimiert werden [81].

Das PRR-System kann auch als Reaktion auf Allergene und Viren aktiviert werden $[82,83]$. PRRs im Epithel sind in der Lage, über Protease-abhängige und Protease-unabhängige Wege Allergenassoziierte Gefahrensignale zu erkennen. Protease-abhängige Signalwege erfordern insbesondere eine direkte Spaltung von Zelloberflächenmolekülen oder die Aktivierung G-Protein-gekoppelter Protease-aktivierter Rezeptoren (PARs) [85-88]. Bei RLRs handelt es sich um zytoplasmatische Rezeptoren, die das Vorhandensein von Viren mit genomischer oder doppelsträngiger RNA bzw. von Zwischenprodukten mit doppelsträngiger RNA aus Viren mit einzelsträngiger RNA erkennen. Melanomdifferenzierung-assoziiertes Gen-5 wird von Epithelzellen in den menschlichen Bronchien exprimiert und ist an der Erkennung von Rhinoviren und anderen Viren beteiligt [89]. NLRs sind intrazelluläre PRRs, die durch eine zentrale Nukleotid-bindende Oligomerisierungsdomäne (NOD) und sich wiederholende Leucin-reiche Sequenzen (leucine-rich repeats) am N-Terminus gekennzeichnet sind [90]. Es sind weitere Arbeiten erforderlich, um die Rolle und Funktion der NOD in Zellen des respiratorischen Epithels zu bestimmen. Auch wenn der Beitrag von Epithelzellen zur antibakteriellen und antiviralen Immunität gut dokumentiert ist, gibt es nur wenige Informationen über die Rolle von Zellen des respiratorischen Epithels in Bezug auf eine Immunität gegen Pilze. CLRs gehören zur Familie der Dectine und erkennen Zellwandkomponenten von Pilzen. Dectin-1 wird beispielsweise von Epithelzellen in den Atemwegen exprimiert und ist für deren Aktivierung durch Mycobacterium, Aspergillus und Allergene wie Hausstaubmilben verantwortlich [91-93]. Dectinrezeptoren können durch $\beta$-Glukane über den TLR4-Signalweg, den TLR2-abhängigen Signalweg oder die Induzierung von oxidativem Stress durch in- trinsische NADPH-Oxidase aktiviert werden [94-96]. Da Pilzsporen starke Allergene darstellen, die in Zusammenhang mit allergischen und chronischen Atemwegserkrankungen wie Asthma stehen, sollte die Bedeutung des Lungenmykobioms nicht vernachlässigt werden [97].

\section{Atemwegsinfektionen und Initiierung der epithelialen Immunantwort}

Infektionen spielen eine zentrale Rolle bei der Induzierung und Exazerbation einer Vielzahl chronischer Atemwegserkrankungen wie Asthma und COPD [98]. Weltweit stellen Infektionen eine Hauptursache für Morbidität und Mortalität dar und können durch Bakterien (z.B. Streptococcus pneumoniae, Haemophilus influenzae, Chlamydia pneumoniae) und Viren (z.B. RSV, Influenza) bedingt sein $[99,100]$. In der Regel wird die Infektion durch eine Exposition des Respirationstrakts gegenüber Bakterienzellen oder Viruspartikeln ausgelöst (Abb. 2).

Bei einer Infektion binden Viren an spezifische zelluläre Rezeptoren, u.a. interzelluläres Adhäsionsmolekül 1 (ICAM-1) und Sialsäurereste, während Bakterien an plättchenaktivierenden Faktor (PAF) und Mannose-6-Phosphat (Man-6-P) binden [101]. Das Wechselspiel zwischen Infektionserregern und Epithelzellen induziert die Stimulation der PRRs sowie die Produktion antimikrobieller Effektormoleküle, Peptide, Enzyme, reaktiver Sauerstoffspezies und einer breiten Palette von Chemokinen und Wachstumsfaktoren. Insgesamt ermöglichen diese Moleküle die Rekrutierung von und Kommunikation zwischen Immunzellen und tragen zur Initiierung der angeborenen Immunantwort bei, die für eine frühe Eindämmung der Infektion von entscheidender Bedeutung ist. Bei Gesunden führt diese Antwort zur umgehenden Elimination der Pathogene. Bei Patienten mit chronischen Atemwegserkrankungen hingegen können diese Abwehrmechanismen gestört sein und zu einer erhöhten Anfälligkeit für Infektionen führen. Diese verstärken ihrerseits die Entzündungsantwort, was wiederum eine Exazerbation der Grunderkrankung nach sich zieht. Bei chronischen Erkrankungen gibt es Belege dafür, dass diese Barrierefunktion gestört ist und es zu einer Auftrennung der Tight Junctions sowie einer erhöhten Permeabilität des Epithels kommt. Dieser Verlust der Barrierefunktion führt zum Eindringen von Pathogenen und anderen Partikeln. Eine gestörte Barrierefunktion steht in engem Zusammenhang mit dem Auslösen anormaler Reparatur- und Remodeling-Prozesse [102]. Atemwegsviren, Luftschadstoffe und Allergene sind allesamt in der Lage, das Epithel auf diese Weise zu schädigen, entweder direkt durch ihre inhärente proteolytische Wirkung oder indirekt durch Bindung an den Rezeptor PAR-2, der auf der apikalen Oberfläche bronchialer Epithelzellen exprimiert wird. So zeigen beispielsweise In-vitro-Studien, dass eine Infektion der Zellen mit Rhinoviren zum Verlust von ZO-1 aus dem Adhäsionskomplex und damit einem reduzierten transepithelialen elektrischen Widerstand (TEER) führt, der auf «Leckagen» in der Barriere hinweist. Sowohl Coxsackie- als auch Rhinoviren beeinträchtigen Occludine [103, 104], während RSV eine Zerstörung der Adhäsi-
246

Kompass Pneumol 2020;8:240-250 DOI: $10.1159 / 000510786$ 


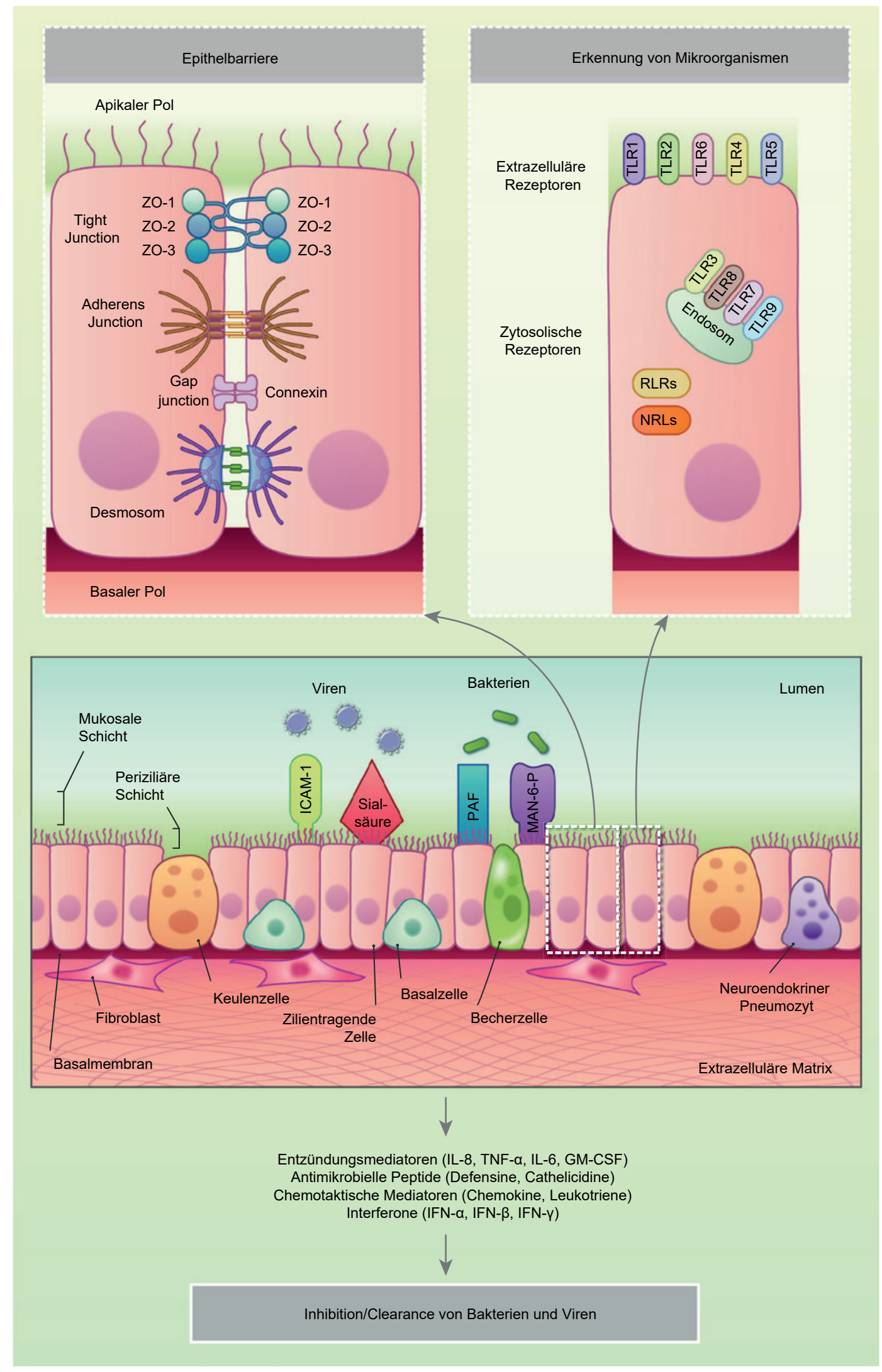

Abb. 2. Reaktion des respiratorischen Epithels auf eine Infektion. Bakterien und Viren binden an zelluläre Rezeptoren, u.a. interzelluläres Adhäsionsmolekül 1 (ICAM-1), Sialsäurereste, plättchenaktivierenden Faktor (PAF) und Mannose-6-Phosphat (Man-6-P). Die Pathogene werden von den Zellen des respiratorischen Epithels internalisiert, was wiederum die Produktion angeborener Immundefensine sowie die Stimulation von intra- und extrazellulären Immunrezeptoren wie Toll-like-Rezeptoren (TLRs), RIG-I-ähnlichen Rezeptoren (RLRs) und NOD-like-Rezeptoren (NLRs) induziert. Bei Gesunden kommt es nach der Generierung einer proinflammatorischen angeborenen bzw. adaptiven Immunantwort zur Elimination der Pathogene. 
onskomplexe verursacht [105]. Bei Asthma wurde in vitro eine Verringerung sowohl von ZO-1 als auch des TEER nachgewiesen, die mit dem Asthma-Schweregrad [106] bzw. E-Cadherin und $\beta$-Catenin korreliert [107]. Des Weiteren wurde bei COPD eine verminderte Expression von Occludinen und Claudinen beobachtet [108]. In vivo wurde nach Influenza-Infektion eine Leckage von Proteinen, z.B. Albumin, in den Alveolarraum berichtet [109]. Viren, Schadstoffe und Allergene stehen bei all diesen Erkrankungen im Zusammenhang mit einer Dysfunktion der ZellZell-Verbindungen und einer Exazerbation der klinischen Symptomatik [103-105, 110, 111].

Defekte in der Barrierestruktur und -funktion wurden in primären Epithelzellkulturen von Asthmatikern selbst nach Vermehrung über mehrere Wochen an einer Luft/Flüssigkeit-Grenzfläche beobachtet, was darauf hindeutet, dass es sich bei der verminderten Barrierefunktion um eine stabile Eigenschaft dieser Zellen handelt [112]. Die Typ-2-Zytokine IL-4 und IL-13 induzieren eine Barrieredysfunktion durch Hemmung der Expression von ZO-1, Occludinen, E-Cadherin und $\beta$-Catenin [113]. Einer These zufolge könnte die Unterbrechung von Zell-Zell-Verbindungen ein Signal für die Stimulierung von Typ-2-T-Helferzellen (Th2) in den Atemwegen darstellen, die Schleimhautintegrität wieder herzustellen [114]. Diese Vorstellung wird durch die Tatsache gestützt, dass ein siRNA-Knockdown bei E-Cadherin zu einer EGFR (epidermaler Wachstumsfaktorrezeptor)-abhängigen Produktion der Th2-stimulierenden Zytokine TARC (thymus- and activation-regulated chemokine) und TSLP (thymic stromal lymphopoietin) durch respiratorische Epithelzellen führt, die den Zusammenhang zwischen Barrieredysfunktion und Th2-gesteuerter Lungenerkrankung erklärt [115]. Kommt der Wundheilungsprozess nach Wiederherstellung der Schleimhautintegrität nicht zum Stillstand, können gesundheitsschädliche gewebliche Umbauvorgänge (Remodeling) in den Atemwegen die Folge sein. E-Cadherin spielt bei Adherens Junctions nicht nur strukturell eine Rolle, sondern ist zudem der Ligand für CD103, das von Immunzellen des angeborenen und adaptiven Immunsystems sowie von einer Subgruppe dendritischer Zellen exprimiert wird. Darüber hinaus bindet es an KLRG1, das von natürlichen Killerzellen, regulatorischen T-Zellen und natürlichen Lymphoidzellen der Gruppe 2 exprimiert wird. Es ist bekannt, dass E-Cadherin und Occludin bei Asthmatikern herunterreguliert sind $[106,116,117]$, und man geht davon aus, dass die Gene für E-Cadherin und Protocadherin-1 in Zusammenhang mit einer Hyperreaktivität der Atemwege stehen [118, 119]. E-Cadherin verankert EGFR in Adherens Junctions [120] und verhindert somit eine Aktivierung des Rezeptors [121, 122]. Infolge der verminderten Expression von E-Cadherin kann EGFR erneut an die luminale Oberfläche der Zelle wandern und dort letztlich aktiviert werden [123]. Eine überschießende Aktivierung von EGFR kann zur Zellproliferation und zur Hyperplasie/Metaplasie von Becherzellen führen [124]. Eine verstärkte Expression von EGFR wurde bei Asthma beobachtet und korrelierte mit einer gestörten Barrierefunktion der Schleimhaut [106].

Wenn $\beta$-Catenin von E-Cadherin dissoziiert ist, kann es sich in den Zellkern verlagern, dort den Wnt-Signalweg aktivieren und so die Zellproliferation induzieren [125]. Dikkopf (DKK)-Proteine regulieren den Wnt-Signalweg über die Interaktion mit Kreme (KRM)-Rezeptoren [126]. Dem Wnt/ $\beta$-Catenin-Signalweg wird eine Rolle bei IPF zugeschrieben [127, 128]. Bei Patienten mit IPF ist die DKK1-Expression in pulmonaler und bronchoalveolärer Lavageflüssigkeit erhöht. Neben ihrer Lokalisation in hyperplastischen Alveolarzellen werden sie am stärksten von Basalzellen des Bronchialepithels exprimiert. Auch die KRM1-Expression ist im Lungengewebe von IPF-Patienten, insbesondere in Bereichen mit Bronchiolisation, erhöht [127]. Die Phosphorylierungssignaturen von $\beta$-Catenin bei IPF sind dieselben wie bei der normalen Lungenentwicklung, was wiederum nahe legt, dass es bei der Reparatur geschädigten Lungengewebes zur Reaktivierung von Entwicklungsprogrammen kommt [129]. Der Wnt/ $\beta$ Catenin-Signalweg spielt außerdem eine pathogenetische Rolle bei COPD, aber in direktem Gegensatz zu den Beobachtungen bei IPF wird der Signalweg im mesenchymalen Kompartiment inaktiviert und nicht aktiviert [130]. Interessanterweise vermutet man, dass als Motor des Krankheitsgeschehens bei IPF eine Verschiebung von dem anerkannten Wnt-Signalweg ( $\beta$-Cateninabhängig) hin zu bislang nicht anerkannten Signalwegen stattfindet [131].

Basalzellen, die als potenzielle Stammzellen in den leitenden Atemwegen fungieren, finden sich in der höchsten Konzentration in der Luftröhre, und ihre Anzahl nimmt in den nachgelagerten Abschnitten des Bronchialbaums in Richtung der Bronchiolen ab. Bei Schädigungen induzieren diese Zellen eine Hochregulierung von p63, einem auf Basalzellen beschränkten Transkriptionsfaktor, der vermutlich bei der epithelial-mesenchymalen Transition (EMT) eine Rolle spielt [132]. Es konnte gezeigt werden, dass bei einer IPF auf fibroblastischen Foki aufliegende epitheliale Basalzellen eine durch die Expression von p63 determinierte gesteigerte Reaktivität entwickeln und einen partiell mesenchymalen Phänotyp annehmen [133] - ein weiterer Beleg für die Beteiligung von ursprünglichen Bronchialzellen bei der Pathogenese der IPF.

Insgesamt ist der Dialog zwischen spezifischen Bakteriengemeinschaften und Wirt ein dynamischer Vorgang. Die Ansiedlung und Lokalisation der Bakteriengemeinschaften in der Lunge werden sowohl durch zelluläre Komponenten in der Lunge wie auch durch abiotische Umgebungsfaktoren (Temperatur, pH-Wert, Mukus und Surfactant) beeinflusst. Insbesondere kann ein Verlust der Barrierefunktion die Zusammensetzung des respiratorischen Mikrobioms dadurch beeinflussen, dass er das Eindringen von Pathogenen und anderen Partikeln ermöglicht. Allerdings ist offensichtlich, dass weitere Daten benötigt werden, um das Wesen des Dialogs zwischen respiratorischer Mikrobiota und Epithelzellen in der Lunge näher zu beschreiben und so die Funktion und Mechanismen, über die das Mikrobiom eine Immunität in der Lunge formt, besser zu verstehen.

\section{Die Darm-Lungen-Achse}

Eine wachsende Zahl an Studien liefert Belege für die Interaktion zwischen Darmflora und Lunge, die sogenannte «Darm-Lungen-
248

Kompass Pneumol 2020;8:240-250 DOI: $10.1159 / 000510786$ 
Achse». Ähnlich wie im Darm wird das Atemwegsmikrobiom der gesunden Lunge von kurzkettige Fettsäuren (SCFA)-produzierenden Bakterien beherrscht: Bacteroidetes, Firmicutes und Proteobacteria. Es handelt sich um stoffwechselaktive Bakterien, die über bakterielle Fermentation unverdaulicher Kohlenhydrate durch das Darmmikrobiom kurzkettige Fettsäuren herstellen. Im menschlichen Intestinaltrakt finden sich von diesen am häufigsten Acetat, Propionat und Butyrat [134]. Mittlerweile ist gut dokumentiert, dass die Darmflora die mikrobielle Zusammensetzung in der Lunge entweder durch direkte bakterielle Besiedlung der Atemwege oder durch Verbreitung kurzkettiger Fettsäuren beeinflusst. Diese kurzkettigen Fettsäuren dienen nicht nur als Brennstoff für die Epithelzellen im Darm, sondern haben auch deutliche physiologische Wirkungen. Sie fungieren als Bindeglied zwischen Mikrobiota und Immunsystem und spielen eine Schlüsselrolle für die Erhaltung der mukosalen Immunität. Auch wenn die Zahl der Studien zur Untersuchung der Wirkungen dieser SCFAs in der Lunge begrenzt ist, haben sich kurzkettige Fettsäuren im Darm als zentral für die Ausbildung von Tight Junctions und die Stimulierung entzündungshemmender Mechanismen zur Aufrechterhaltung der intestinalen Homöostase erwiesen [135]. Tatsächlich interagieren kurzkettige Fettsäuren mit Immunzellen und modulieren deren Rekrutierung, Differenzierung, Aktivierung und Überlebensfähigkeit in unterschiedlichen Geweben [136]. Der vorgeschlagene Mechanismus dieser Darm-Lungen-Interaktion beinhaltet die Einwanderung der Mikrobiota und ihrer Produkte aus den Atemwegen in die Darmschleimhaut. Dort werden sie phagozytiert und von antigenpräsentierenden Zellen, die das Priming von B- und T-Zellen anregen, zu den mesenterialen Lymphknoten weitergeleitet. Sobald sie aktiviert sind, können diese Zellen dann zurück an ihren Ursprungsort oder in distal gelegene Regionen, etwa in das Lungenepithel, wandern, wo sie direkt auf die Zielgewebe wirken können. Ein weiterer vorgeschlagener Weg involviert die direkte Einwanderung bakterieller Produkte aus der Darmschleimhaut über die Blutbahn in die Lunge, wo sie eine Stimulierung des Immunsystems bewirken. Es wird vermutet, dass derselbe Weg auch in umgekehrter Richtung, also beginnend in der Lunge und in der Darmschleimhaut endend, existiert, auch wenn der Einfluss der Lungenmikrobiota und ihrer Metaboliten auf den Darm noch ungeklärt ist [137].

Es hat sich erwiesen, dass die Zusammensetzung einer stabilen Darmflora einen Einfluss auf die Lungenimmunität hat. Bei Mäusen, denen im frühen Lebensalter die Darmmikrobiota fehlt, ist das Risiko für allergische Atemwegserkrankungen erhöht [138, 139]. Ein erhöhtes Asthmarisiko wird mit einem vorherrschenden Auftreten von Bacteroidetes fragilis und anaeroben Mikroben insgesamt in der frühen Lebensphase [140] und einer Verminderung der Gesamtvielfalt der Bakteriengattungen Escherichia coli, Faecalibacterium, Lachnospira, Rothia und Veillonella in Zusammenhang gebracht [141]. Bei Erwachsenen wurden in der Darmflora taxaspezifische Unterschiede beobachtet, beispielsweise ein relativ erhöhtes Vorkommen von Bifidobacterium adolescentis, welches mit der Zeit seit der Asthma-Diagnose negativ korrelierte [142]. Des Weiteren reduzierte bei Mäusen eine peptidbasierte enterale Ernährung, die die Produktion kurzkettiger Fettsäuren anregte, eine Elastase-induzierte Entzündung und Emphysem in der Lunge [143]. Die Achse ist mitnichten eindirektional: So begünstigen Atemwegsinfektionen Störungen im Darmmikrobiom, aber weitere Arbeiten sind erforderlich, um dies zu validieren $[144,145]$. Laut dem Bericht einer Studie erschien ein nicht resorbierbarer Tracer nach intranasaler Verabreichung in die Nasenhöhle von Mäusen kurze Zeit später im Gastrointestinaltrakt [144]. Darüber hinaus berichten Sze et al. [146], dass eine intratracheale Verabreichung von Lipopolysaccharid nicht nur Störungen im Atemwegsmikrobiom verursachte, sondern auch zur Translokation von Atemwegsbakterien der Gattungen Clostridium und Lachnospiraceae in die Blutbahn und innerhalb von 24 Stunden zu Wirkungen auf die Darmmikrobiota führte, was einen deutlichen Anstieg der Gesamtbakterienlast nach sich zog. Insgesamt liefern diese Studien Belege für die bidirektionale Kommunikation zwischen den beiden Organsystemen. Allerdings steckt unser Verständnis dieses Dialogs noch in den Kinderschuhen. Es ist dringend notwendig zu klären, ob diese Veränderungen der Mikrobiota Ursache oder Wirkung der Erkrankung sind. Des Weiteren gibt es immer mehr Belege, die die Rolle oraler Antibiotika und ihre Wirkung auf die Lungenimmunität unterstreichen. Antibiotika könnten das Lungenmikrobiom über ihre Wirkungen auf das Darmmikrobiom und nachfolgend auf die Lungenimmunität entweder direkt oder indirekt beeinflussen. Zur Stützung dieser Hypothese zeigen Untersuchungen an Mäusen, dass eine Elimination bestimmter Arten aus dem Darmmikrobiom infolge der Einnahme von Antibiotika Einfluss auf Lungenerkrankungen und allergische Entzündungen hat [147]. So korrelierte eine mikrobielle Dysbiose bedingt durch die Anwendung von Makrolidantibiotika in den ersten Lebensjahren bei finnischen Vorschulkindern mit einem erhöhten Risiko für Asthma [148]. Ohne Zweifel müssen wir die Interaktionen zwischen Darm- und Atemwegsmikrobiom anerkennen, um die Wirkungen von Antibiotika auf die Lungengesundheit verstehen zu können.

\section{Schlussfolgerungen und Zukunftsperspektiven}

Man geht mittlerweile davon aus, dass das Oberflächenepithel der Lunge, einschließlich der oberen und unteren Atemwege, eine Schlüsselrolle bei der Aufrechterhaltung der Immunhomöostase spielt. Das Epithel fungiert als erste Verteidigungslinie gegen eingeatmete virale, bakterielle und mykotische Krankheitserreger. Studien belegen, dass das Oberflächenepithel des menschlichen Respirationstrakts von einer komplexen und dynamischen Mikrobiota besiedelt ist, die sowohl in Gesundheit als auch in Krankheit eine wichtige Rolle spielt. Zunehmende Anerkennung findet die Auffassung, dass Etablierung und Persistenz dieser bakteriellen Gemeinschaften durch die zellulären und physiologischen Eigenschaften der Lunge beeinflusst werden. Eine Dysregulation der epithelialen Immunantwort und Barrierefunktion kann in Kombination mit einer mikrobiellen Dysbiose chronisch-entzündliche Lungenerkrankungen wie Asthma, COPD, CF und IPF 
begünstigen. Allerdings ist die genaue Art der Beziehung zwischen respiratorischem Mikrobiom und Lungenepithel nach wie vor Gegenstand aktiver Forschung. Ein besseres Verständnis dieses Wechselspiels in Bezug auf die Erhaltung einer Atemwegshomöostase wird neue Einblicke in die Pathogenese verschiedener Atemwegserkrankungen ermöglichen sowie neue Chancen für Diagnostik und Therapie eröffnen. Neue Evidenzdaten, insbesondere zu kurzkettigen Fettsäuren (SCFAs), weisen eindeutig auf eine Verbindung zwischen Darm und Lunge sowie der dort vorkommenden mikrobiellen Gemeinschaften hin. Die Ergebnisse unterstreichen die Notwendigkeit künftiger Studien zur näheren Beschreibung der Mikrobiota und Metaboliten dieser beiden Schleimhautumgebungen und zur Bestimmung ihrer Interaktionen mit dem Wirtsgewebe, um gezieltere Behandlungsansätze für Lungenerkrankungen entwickeln zu können.

\section{Disclosure Statement}

Die Autoren erklären, dass keine konkurrierenden Interessen oder Interessenskonflikte bestehen.

\section{Literatur}

Die Literatur ist unter https://www.karger.com/Article/Fulltext/510786 abrufbar.

\section{Lizenzangabe}

Rachele Invernizzi, Clare M. Lloyd, Philip L. Molyneaux: Respiratory microbiome and epithelial interactions shape immunity in the lungs. Immunology. 2020;160(2):171-182. ${ }^{\circ} 2020$ The Author(s) (Übersetzung), lizensiert unter CC BY 4.0 (https://creativecommons.org/licenses/by/4.0/deed.de). 